\title{
Static and dynamic structure of liquid metals: Role of the different parts of the interaction potential
}

\author{
M. Canales \\ Department de Física i Enginyeria Nuclear, Universitat Politècnica de Catalunya, Campus Nord, Sor Eulàlia d'Anzizu, B4-B5, 08034 \\ Barcelona, Spain \\ J. A. Padró \\ Departament de Física Fonamental, Universitat de Barcelona, Diagonal, 647, 08028 Barcelona, Spain
}

(Received 10 February 1997)

\begin{abstract}
The influence of different parts of the interaction potential on the microscopic behavior of simple liquid metals is investigated by molecular dynamics simulation. The role of the soft-core repulsive, short-range attractive, and long-range oscillatory forces on the properties of liquid lithium close to the triple point is analyzed by comparing the results from simulations of identical systems but truncating the potential at different distances. Special attention is paid to dynamic collective properties such as the dynamic structure factors, transverse current correlation functions, and transport coefficients. It is observed that, in general, the effects of the short-range attractive forces are important. On the contrary, the influence of the oscillatory long-range interactions is considerably less, being the most pronounced for the dynamic structure factor at long wavelengths. The results of this work suggest that the influence of the attractive forces becomes less significant when temperature and density increase. [S1063-651X(97)13108-7]

PACS number(s): 61.20.-p
\end{abstract}

\section{INTRODUCTION}

A detailed knowledge of the role played by the different parts of the interatomic potential on the microscopic behavior of liquids is of great interest for understanding the basis of the liquid state properties and a useful guide for obtaining more refined potential models. This sort of information cannot be deduced from experimental measurements on real systems but can be obtained from suitable computer simulation "'experiments.', Molecular dynamics (MD) simulation is one of the most useful tools for this kind of investigation. MD studies about the influence of the repulsive and attractive forces on the microscopic properties of dense simple liquids were carried out since the early stages of the development of computer simulation. However, the vast majority of these studies were devoted to structural and single-particle dynamical properties [1-7]. To our knowledge, the only papers about the effects of the different part of potentials on the dynamic collective properties of simple liquids are a paper on the dispersion of sound modes in liquid $\mathrm{Rb}$ and LennardJones (LJ) fluids [8] and two recent papers on soft-sphere fluids [9] and hard-core fluids with a Yukawa tail [10].

The aim of this paper is to analyze the influence of the different parts of the interaction potential on the properties of liquid metals close to the triple point, paying special attention to the dynamic collective properties. To this end, the MD results using the full potential (in practice, with a relatively long cutoff) are compared with those for identical systems but truncating the potential at shorter interatomic distances. The typical interionic potential functions for liquid metals show a soft repulsive wall, a deep attractive well, and a long-range oscillatory tail. Potential functions truncated either at the first minimum or maximum have been considered in this work. The former correspond to soft-core potentials whereas the latter include both the soft-core and the short- range attractive parts but not the long-range oscillations.

MD simulations of $\mathrm{Li}$ atoms have been carried out by assuming two effective pair potential models. The former (LM1) is a potential with no adjustable parameters deduced from the neutral pseudoatom method [11]. Both structural and dynamical properties resulting from MD simulations using this model are in satisfactory agreement with the available experimental data for liquid Li [12-14]. Potentials for $\mathrm{Li}$ alloys (Li-Mg, Li-Na) deduced by the same method reproduce satisfactorily the experimental structure of these systems [15]. The second potential (LM2) [13] was obtained by assuming an empty core pseudopotential with a core radius determined by fitting the calculated main peak of the static structure factor to the experimental value. These two potentials show marked differences. The attractive well of LM2 is shallower and located at higher $r$ than that of LM1 (the two potentials are compared in Fig. 1 of Ref. [13]). For a simple characterization of the potential functions two parameters are ordinarily used, i.e., the position of the first zero $(\sigma)$ and the depth of the first minimum $(\epsilon)$. The $\sigma$ and $\epsilon$ parameters corresponding to LM1 and LM2 are gathered in Table I. Since the effective atomic size for the second is larger, the atomic

TABLE I. Length and energy parameters $(\sigma, \epsilon)$ corresponding to different potentials. Reduced densities and temperatures $\left(\rho^{*}, T^{*}\right)$ for the thermodynamic state considered in this work $(\rho=4.4512$ $\left.\times 10^{-2} \AA^{-3} ; T=470 \mathrm{~K}\right)$.

\begin{tabular}{lrrrr}
\hline \hline Potentials & $\sigma(\AA)$ & $\epsilon(\mathrm{K})$ & $\rho^{*}$ & $T^{*}$ \\
\hline LM1; RLM1; TLM1 & 2.57 & 887 & 0.756 & 0.530 \\
LM2; RLM2; TLM2 & 2.95 & 248 & 1.143 & 1.893 \\
Torcini et al. $[16]$ & 2.68 & 582 & 0.860 & 0.808 \\
Novotny et al. $[18]$ & 2.64 & 750 & 0.819 & 0.627 \\
Kambayashi et al. $[19]$ & 2.64 & 1050 & 0.819 & 0.448 \\
\hline \hline
\end{tabular}


cores are notably closer. A quantitative measure of the atomic close packing is given by the fraction $\eta \equiv \rho \pi \sigma^{3} / 6$. The packing fraction for LM1 $(\eta=0,4)$ is markedly smaller than that for LM2 $(\eta=0,6)$. It should be also noticed that for LM1, $\epsilon>k_{B} T$ while for LM2, $\epsilon<k_{B} T$. Despite their marked differences, the majority of the properties obtained from LM1 and LM2 do not show considerable discrepancies [13]. This fact will be the subject of discussion in this paper. We want to point out that a careful comparison of the MD results with experimental findings indicates that simulations using LM1 are more realistic than those using LM2 [13,14].

Two effective pair potentials somewhat different from those considered in this work have been used in recent MD simulations of liquid Li. Torcini et al. [16] adopted the potential implemented by Price et al. [17] that considers an empty core pseudopotential adjusted to reproduce the microscopic properties in the solid phase. Nowotny et al. [18] proposed a potential based on a nonlocal pseudopotential and Kambayashi et al. [19] constructed a potential according to the quantal hypernetted-chain theory. These potentials also produce results in reasonable accordance with experimental data $[16,18,19]$ and the corresponding $\sigma$ and $\epsilon$ parameters, are markedly closer to those for LM1 than to those for LM2 (see Table I). The reduced densities $\left(\rho^{*}=\rho \sigma^{3}\right)$ and temperatures $\left(T^{*}=k_{B} T / \epsilon\right)$ are useful quantities to compare the properties of different systems. Discussions on the possible application of the corresponding states picture to the analysis of the properties of liquid metals go back to the 1970s [20]. Balucani et al. [21] found that several liquid alkali metals ( $\mathrm{Na}, \mathrm{K}, \mathrm{Rb}, \mathrm{Cs}$ ) at states close to the melting point show a scaling behavior, i.e., when reduced units are used, the potentials by Price et al. [17] for liquid alkali metals nearly coincide and both structural and time-dependent properties show common features. The reduced temperature and density for the liquid metals considered by Balucani et al. $\left(\rho^{*}\right.$ $\left.=0.895, T^{*}=0.78-0.84\right)$ are intermediate between those for LM1 and LM2, though noticeably closer to those for LM1 (Table I). According to these findings, the LM1 and LM2 potentials may be considered as two extreme models of liquid alkali metals near the melting point, though MD simulations using LM1 are closer to actual systems and the resulting properties may be taken as being representative of simple liquid metals close to the triple point. Moreover, findings using LM2 provide information on the tendency of properties when $T$ and $\rho$ increase.

\section{MD SIMULATIONS DETAILS}

We carried out MD simulations of systems made up of 668 atoms with the mass of ${ }^{7} \mathrm{Li}$ enclosed in a box with periodic boundary conditions. The density and temperature of the simulated systems $\left(\rho=4.4512 \times 10^{-2} \AA^{-3} ; \quad T\right.$ $=470 \mathrm{~K}$ ) correspond to liquid ${ }^{7} \mathrm{Li}$ near to the triple point. The properties were calculated from the configurations generated over runs of about $10^{5}$ time steps. The time step was of $3 \mathrm{fs}$. The $k$-dependent properties were calculated for ten different $k$ values between 0.25 and $4.08 \AA^{-1}$.

The potentials were truncated at $r_{c}=9.25 \AA$ and $r_{c}$ $=10.62 \AA$ in the simulations corresponding to the full LM1 and LM2 potentials, respectively. In the simulations with the purely repulsive cores (RLM1 and RLM2) the potentials were truncated at their first minima $r_{c}=3.05 \AA$ and $r_{c}$ $=3.30 \AA$, respectively. In the simulations with the truncated potentials including a repulsive core and an attractive well (TLM1 and TLM2), the cutoffs were at the position of the first maxima $r_{c}=4.75 \AA$ and $r_{c}=4.66 \AA$, respectively.

\section{STRUCTURE AND SINGLE PARTICLE DYNAMICS}

It is generally accepted that "geometric' factors associated with the packing of atoms have a dominant influence on the structure of dense simple liquids, which is largely determined by the repulsive part of the pair interactions. This fact is on the basis of the perturbation theories that have been successfully applied to the study of the equilibrium structural and thermodynamic properties of different liquids [22,23]. The influence of the repulsive forces on the structure of liquid metals has been recently analyzed by integral equation calculations. Matsuda et al. [24] observed that the radial distribution function $[g(r)]$ of liquid Cs is considerably influenced by the part of the potential beyond its first minimum; especially important is the contribution of the short-range attractive part, which enhances the oscillations of $g(r)$ but does not modify their location. Similar findings were obtained by Bretonnet and Jakse [25] for expanded liquid Rb and Cs. The $g(r)$ 's resulting from our simulations (Fig. 1) corroborate these conclusions. The maxima and minima of the $g(r)$ for TLM1 are more marked than for RLM1 whereas the $g(r)$ for LM1 and TLM1 are quite close and the only noticeable difference is a slight shift of the oscillations after the first maximum. The effects of the short-range attractive forces in LM2 and TLM2 are markedly smaller. As may be observed in Fig. 1 the $g(r)$ for RLM2 is very close to that for LM1 (it should be noted that the $g(r)$ 's for LM2 and LM1 are almost identical [13]). The differences between the $g(r)$ 's corresponding to LM2 and TLM2 are still smaller than those between LM1 and TLM1.

We have also determined the statistical distributions of the bond angle between a central particle and particles in both the first and the second coordination shells [26]. It is expected that these functions can be more sensitive to the details of potentials since they are directly related to the three-body correlation functions. Nevertheless, the results for LM1 and LM2 do not show considerable discrepancies [13]. Moreover, the influence of the different parts of the potential on the bond-angle distributions is not significantly different from that on the $g(r)$. For the sake of brevity these distributions are not reported in this paper.

The resulting velocity autocorrelation functions $[C(t)]$ are displayed in Fig. 1. As with $g(r)$, the $C(t)$ functions corresponding to LM1 and LM2 are very close and only the former is shown in Fig. 1. According to earlier results of Schiff [2], we can observe that the changes in $C(t)$ when the potential is truncated at its first maximum are very small. However, the $C(t)$ negative region is considerably reduced when the attractive forces are left out. The discrepancies between the $C(t)$ 's for TLM1 and RLM1 are much more important than those for TLM2 and RLM2. The self-diffusion coefficients $(D)$ are proportional to the time integral of the $C(t)$ functions. The qualitative dependence of the resulting $D$ coefficients (Table II) on the different parts of the potential is consistent with that observed for the $C(t)$ 's. 

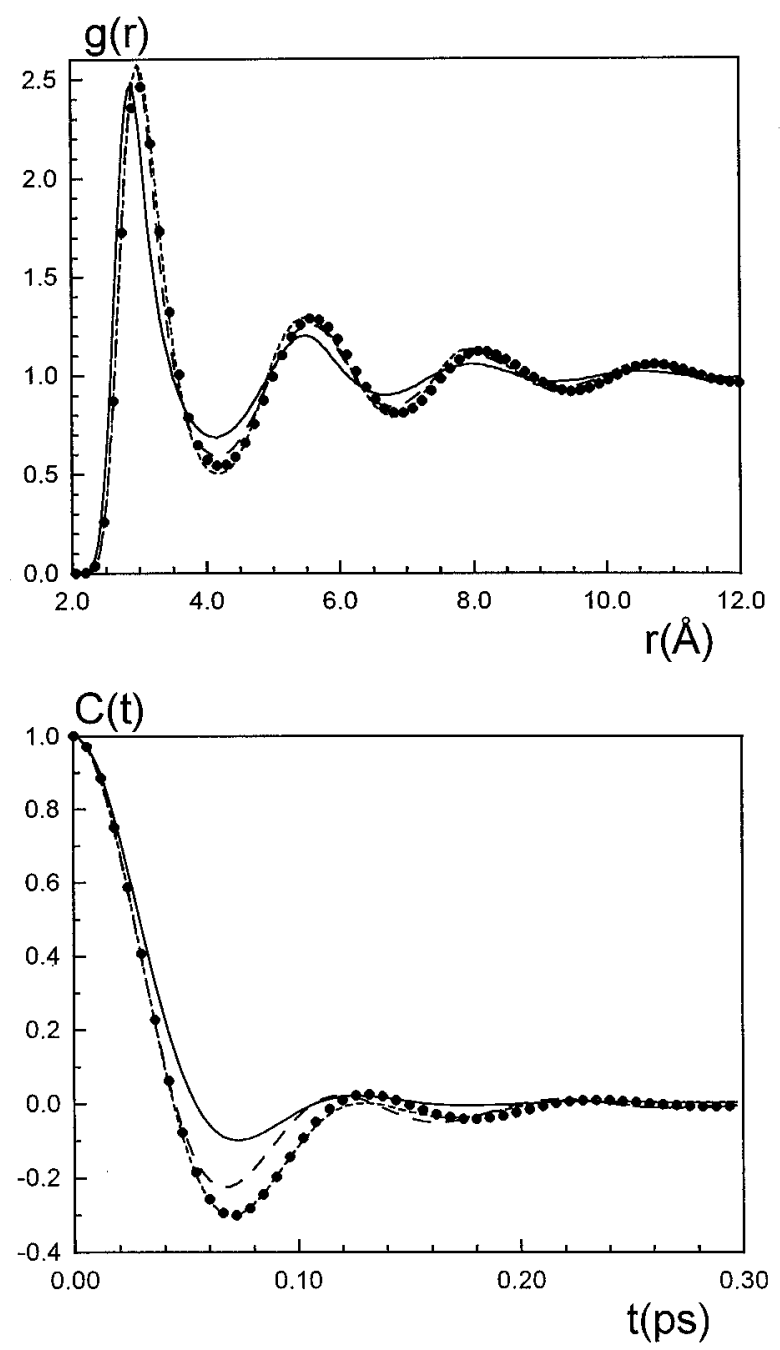

FIG. 1. Radial distribution functions (up) and normalized velocity autocorrelation functions (down). Solid circles, LM1 potential; short-dashed lines, TLM1 potential; solid line, RLM1 potential; long-dashed lines, RLM2 potential.

The results discussed in this section corroborate that both $g(r)$ and $C(t)$ of liquid metals are noticeably influenced by the attractive part of the first well of the potential. This influence, which can be more clearly observed in the case of $C(t)$, is much more marked for LM1 than for LM2 (it should be noted that the former model may be considered as representative of liquid metals close to the triple point). However, the oscillatory tail of the potential has a rather small influence on $g(r)$ and $C(t)$ although it can have considerable effects on the low- $k$ limit of the structure factor (see Sec. IV B). If one compares the results for RLM1 and RLM2, one observes that the former produces a less structured $g(r)$ and a $C(t)$ with less pronounced negative values as corresponds to a system with a lower close-packing fraction. Nevertheless, as a result of the strong attractive forces in LM1 these differences vanish when the full potentials are considered.

\section{DYNAMIC COLLECTIVE PROPERTIES}

\section{A. Transport coefficients}

The Green-Kubo relations have been used for the calculation of various transport coefficients. According to these relations the shear viscosity $\left(\eta_{S}\right)$, longitudinal viscosity $\left(\eta_{L}\right)$, and thermal conductivity $(\lambda)$ coefficients are proportional to the time integral of the correlations of the nondiagonal and diagonal elements of the stress tensor and the energy current, respectively [23,27]. These time correlation functions decay monotonically to zero, as may be observed in Fig. 2 for the functions corresponding to $\eta_{S}$ and $\lambda$. The resulting coefficients are reported in Table II.

The long-range oscillatory tail of the potential has a rather small influence on the values of the viscosity coefficients. However, the contribution of the short-range attractive forces to these properties is more important, especially in the case of LM1 and TLM1. When the attractive part of TLM1 is removed we can observe a substantial increase of $\eta_{S}$ and $\eta_{L}$ whereas in the case of TLM2 the changes are qualitatively the same but markedly smaller. We can observe small but noticeable contributions to $\lambda$ of both the attractive and oscillatory parts of LM1, whereas these contributions are appreciably smaller for LM2. It is interesting to note that the different values of $\lambda$ for LM1, TLM1, and RLM1 correspond to the integral of very close functions. As with other properties, the transport coefficients as well as the corresponding time correlation functions for LM1 and LM2 do not show noticeable discrepancies [13].

\section{B. Dynamic structure factor}

The spectrum of the density fluctuations $[S(k, \omega)]$, usually called dynamic structure factor, is directly related to the

TABLE II. Summary of the results from the MD simulations using different potential models.

The definitions of properties and potentials are given in the text.

\begin{tabular}{lllllll}
\hline \hline & LM1 & TLM1 & RLM1 & LM2 & TLM2 & RLM2 \\
\hline$D\left(10^{-9} \mathrm{~m}^{2} \mathrm{~s}^{-1}\right)$ & 6.6 & 5.9 & 16.0 & 6.3 & 6.3 & 9.0 \\
$S(0)\left(10^{-2}\right)$ & 2.5 & 4.5 & 3.0 & 2.0 & 2.0 & 2.0 \\
$k_{L}^{\max }\left(\AA^{-1}\right)$ & 1.84 & 1.84 & 0.88 & 1.84 & 1.84 & 1.44 \\
$k_{T}^{\min }\left(\AA^{-1}\right)$ & $0.25^{\mathrm{a}}$ & $0.25^{\mathrm{a}}$ & 1.02 & $0.25^{\mathrm{a}}$ & $0.25^{\mathrm{a}}$ & 0.44 \\
$k_{T}^{\max }\left(\AA^{-1}\right)$ & $4.08^{\mathrm{b}}$ & $4.08^{\mathrm{b}}$ & 1.84 & $4.08^{\mathrm{b}}$ & $4.08^{\mathrm{b}}$ & 4.08 \\
$\eta_{s}\left(10^{-4} \mathrm{~Pa} \mathrm{~s}\right)$ & 5.1 & 6.5 & 1.9 & 5.8 & 5.3 & 3.4 \\
$\eta_{L}\left(10^{-4} \mathrm{~Pa} \mathrm{~s}\right)$ & 8.5 & 8.6 & 3.2 & 8.6 & 8.4 & 5.6 \\
$\lambda\left(\mathrm{J} \mathrm{m}^{-1} \mathrm{~s}^{-1} \mathrm{~K}^{-1}\right)$ & 1.3 & 1.1 & 0.8 & 1.3 & 1.2 & 1.2 \\
\hline \hline
\end{tabular}

${ }^{\mathrm{a}} k_{T}^{\min }$ may be smaller than $0.25 \AA^{-1}$, which is the lowest $k$ value in this work.

${ }^{\mathrm{b}} k_{T}^{\max }$ may be greater than $4.08 \AA^{-1}$, which is the highest $k$ value in this work. 

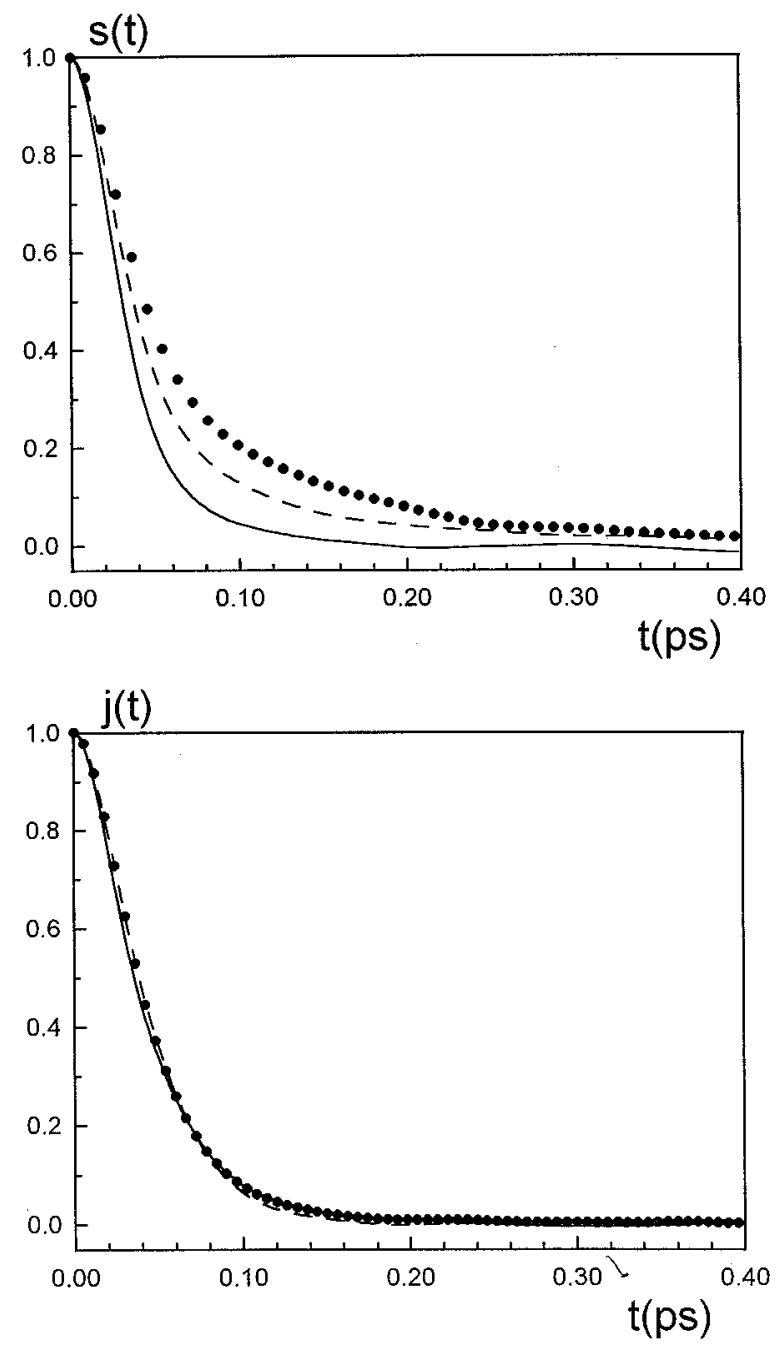

FIG. 2. Normalized time correlation functions of the offdiagonal stress tensor elements (up) and the energy current (down). Solid circles, LM1 potential; solid line, RLM1 potential; longdashed lines, RLM2 potential.

spectrum of the longitudinal-current fluctuations [23,28]. At large $k$ values (kinetic regime), $S(k, \omega)$ approaches its free particle expression and, for a given $k$, has a Gaussian shape. At short $k$ values (hydrodynamic regime), $S(k, \omega)$ approaches the expression for a continuum and, for a given $k$, consists of three Lorentzian lines, the Rayleigh line at $\omega$ $=0$ and two shifted Brillouin lines. The presence of the Brillouin peaks reflects the propagation of density fluctuations (longitudinal modes) and they disappear when the $k$ values approach those for the kinetic regime. The maximum value of $k$ for which $S(k, \omega)$ show noticeable Brillouin peaks $\left(k_{L}^{\max }\right)$ has been determined for each system and the results are listed in Table II [it should be pointed out that we cannot determine $k_{L}^{\max }$ very accurately since $S(k, \omega)$ was only calculated for ten $k$ values, i.e., those in Fig. 3 and $k=0.51$, $0.88,1.25$, and $\left.1.44 \AA^{-1}\right]$. A correlation between $k_{L}^{\max }$ and $\eta_{L}$ can be observed, so that liquids with higher viscosity can sustain propagating longitudinal waves up to higher wave numbers. The persistence of the Brillouin peaks as $k$ increases was related to the softness of the interaction potential and, consequently, to the isothermal compressibility of the
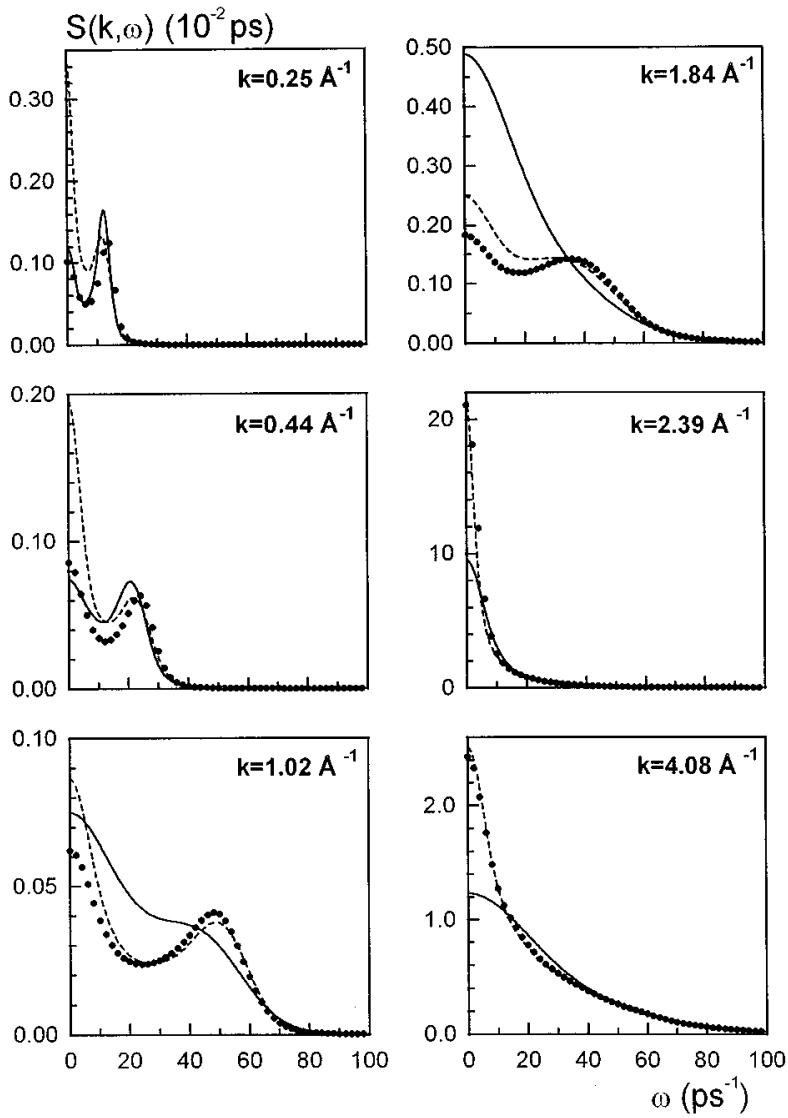

FIG. 3. Dynamic structure factors. Solid circles, LM1 potential; short-dashed lines, TLM1 potential; solid line, RLM1 potential.

liquid [23]. However, the results of this work show that the attractive forces also play a significant role in the propagation of longitudinal modes in liquid metals. So, $k_{L}^{\max }$ for LM1 is markedly different from that for RLM1 despite the softcore repulsive part in the two potentials being the same. In the case of LM2, the influence of the attractive forces is less important. According to our results the long-range oscillatory tail does not have any noticeable influence on $k_{L}^{\max }$. Moreover, we cannot observe any significant correlation between the $k_{L}^{\max }$ values for LM1, TLM1, RLM1 and $S(0)$ (Table II) that is proportional to the isothermal compressibility coefficient.

The $S(k, \omega)$ results for LM1, TLM1, and RLM1 are shown in Fig. 3. At small $k, S(k, \omega)$ for LM1 is quite close to that for RLM1 but markedly different for $S(k, \omega)$ for TLM1, which shows a considerably higher Rayleigh peak. These findings indicate that, in the hydrodynamic region, the contributions to $S(k, \omega)$ of both the short-range attractive part and the oscillatory tail of potential are important, are in opposite directions, but do not affect significantly the location of the Brillouin peaks. At large $k$, the influence of the potential tail is almost negligible whereas the short-range attractive forces produce a considerable increase of the $S(k, 0)$. $S(k, \omega)$ for LM2 (that is not reported in this paper but is shown in Fig. 11 of Ref. [13]) does not show significant changes when the attractive and oscillatory tails of the potential are removed. The discrepancies between the $S(k, \omega)$ results for different systems (e.g., between LennardJones and liquid metals) were attributed to the different soft- 


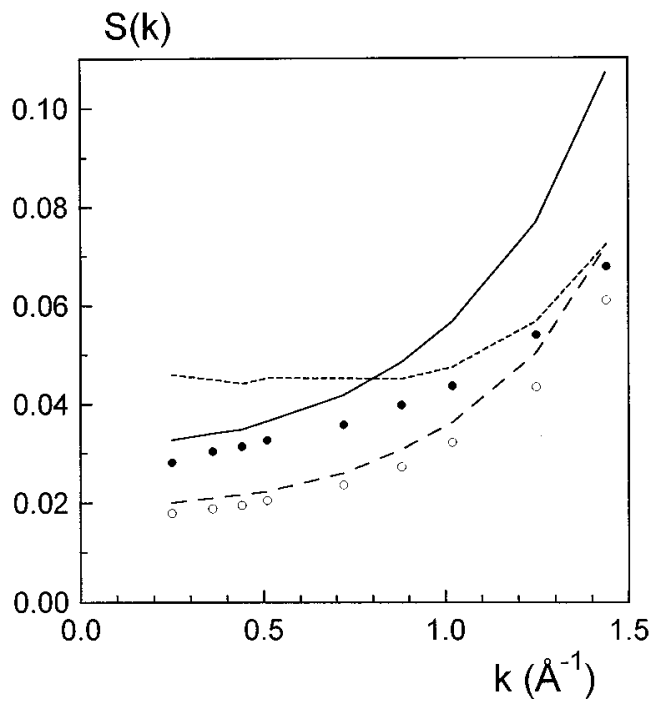

FIG. 4. Static structure factors in the small- $k$ region. Solid circles, LM1 potential; short-dashed lines, TLM1 potential; solid line, RLM1 potential; open circles, LM2 potential; long-dashed lines, RLM2 potential.

ness of the core repulsion $[9,23]$. However, the MD results of Ebbsjö et al. [29] for liquid Al using three potential models suggested that the contribution of the attractive part of the potential may be important in the case of liquid metals. Our findings corroborate the results of Ebbsjö et al.

The static structure factor $S(k)$ is ordinarily obtained as the Fourier transform of $g(r)$. However, at small $k$ it is more convenient to determine the intermediate scattering function $F(k, t)$ and make use of the relation $F(k, 0)=S(k)$ [13]. The resulting $S(k)$ in the low- $k$ region and the corresponding extrapolated $S(0)$ values are shown in Fig. 4 and Table II, respectively. As with other properties, the results for LM2 show little changes when different cutoffs of the potential are used whereas the results for LM1, RLM1, and TLM1 show noticeable discrepancies. It is interesting to note that the short-range attractive and the long-range oscillatory part of LM1 produce similar changes but in the opposite direction on $S(0)$. These results are in accordance with those from integral equation calculations [24,25], which showed that the low- $k$ limit of $S(k)$ in liquid metals at low densities is strongly dependent on the cutoff distance for the interaction potential. As in this work, it was observed that the attractive forces increase $S(0)$. This is physically reasonable since $S(0)$ is proportional to the isothermal compressibility coefficient.

\section{Transverse current correlation functions}

The power spectra of the transverse-current correlation functions $\left[C_{T}(k, \omega)\right]$ provide information on how the system responds to shear disturbances with wave vector $k$ and frequency $\omega$. In the kinetic regime, $C_{T}(k, \omega)$ approaches the free particle expression and, for a given $k$, has a Gaussian shape. In the hydrodynamic regime, $C_{T}(k, \omega)$ approaches the expression for a continuum and, for a given $k$, has a Lorentzian form. In these extreme regimes the fluid cannot support the propagation of transverse modes but MD findings have shown that, in intermediate $k$ ranges, $C_{T}(k, \omega)$ for a given
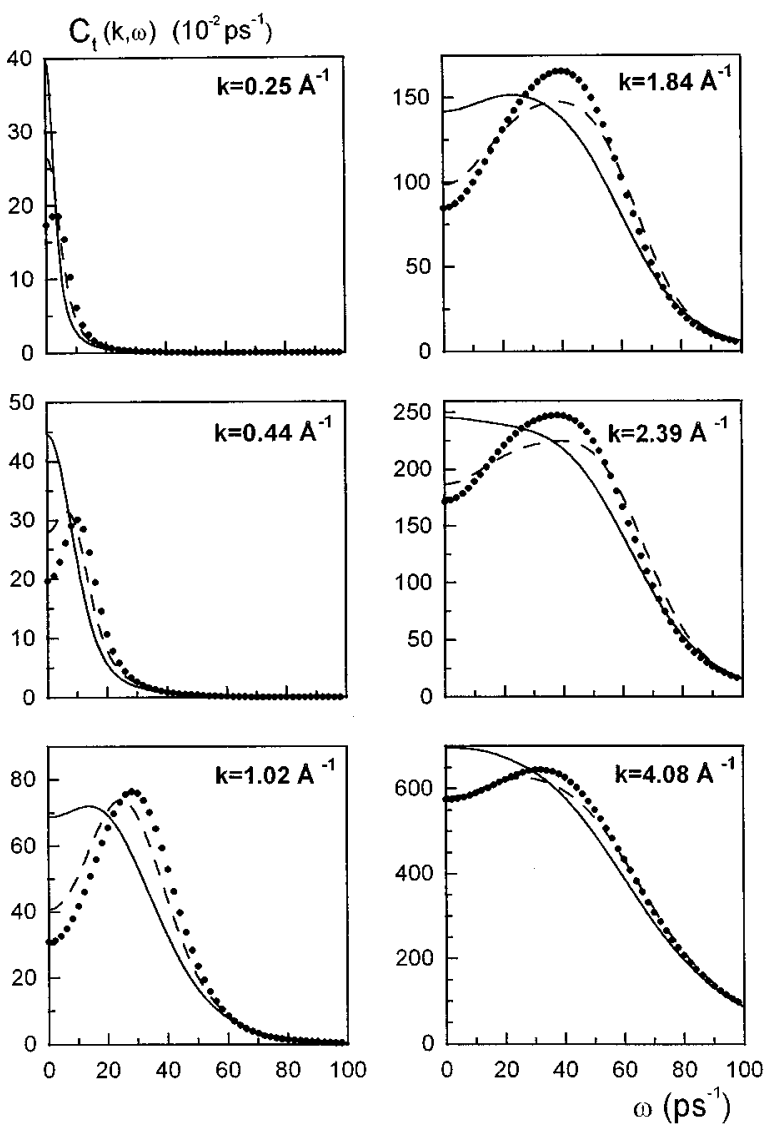

FIG. 5. Transverse current correlation functions. Solid circles, LM1 potential; solid line, RLM1 potential; long-dashed lines, RLM2 potential.

$k$ can show a peak at nonzero frequency that can be associated with propagating shear waves. The appearance of shear waves can be accounted for by the incorporation of viscoelastic effects into the hydrodynamic model for the transverse current $[23,28]$. For the systems in this work we have determined a $k$ interval for which $C_{T}(k, \omega)$ shows a noticeable peak. The extreme $k$ values of these intervals $\left(k_{T}^{\text {min }}\right.$ and $k_{T}^{\max }$ ) are gathered in Table II. As may be expected, the propagation of transverse waves and the shear viscosity coefficients are correlated and the narrower $k$ intervals correspond to the systems with lower $\eta_{s}$.

We have not observed any significant influence of the long-range oscillatory forces on $C_{T}(k, \omega)$. On the contrary, the effects of the short-range attractive forces may be important. So, the $C_{T}(k, \omega)$ curves for LM1 and RLM1 show large discrepancies. The $C_{T}(k, \omega)$ 's for RLM1 show higher initial values and maxima at lower $\omega$ than for LM1. Moreover, for RLM1 we can only observe a maximum for a narrow interval of $k$ whereas for LM1 a maximum can be observed for all the values of $k$ considered in this work (Table II). In the case of LM2 the influence of the short-range attractive forces is clearly smaller and we have only found noticeable discrepancies between the $C_{T}(k, \omega)$ results for LM2 and RLM2 at the lowest $k\left(0.25 \AA^{-1}\right)$. It should be pointed out that the results for LM2 have not been represented in Fig. 5 since they are almost identical to those for LM1 [13].

The $S(k, \omega)$ and $C_{T}(k, \omega)$ findings for RLM1 and TLM1 show that the contribution of the short-range attractive forces 
to the propagation of both longitudinal and shear waves in liquid metals is important. However, the results for RLM2 and TLM2 show that the effect of attractive forces in systems at high packing fractions is screened out and the propagation of longitudinal and shear waves should be mainly associated with the almost continuous collisions among the close atomic cores.

\section{CONCLUDING REMARKS}

The influence of the different parts of the potential on the collective dynamic properties of the systems analyzed in this paper is qualitatively similar to that observed for the structure and single dynamic properties. The results for the LM1 potential using different cutoffs indicate that the collective dynamics properties of simple liquid metals close to the triple point are considerably influenced by the short-range attractive forces corresponding to the first well of the effective pair potentials (which are very deep for these systems). However, the effects of the long-range oscillatory interactions corresponding to interatomic distances beyond the first maximum of the potential are considerably smaller. The only significant contribution of these interactions has been for
$S(k, \omega)$ at small $k$ values and, consequently, for $S(0)$ and the isothermal compressibility. In the case of LM2, the influence of the part of the potential beyond the first minimum is markedly smaller than for LM1. As was commented in the Introduction of this paper, MD simulations with LM2 correspond to a system at higher reduced density and temperature than those for LM1 (see Table I). So, the results presented in this work suggest that the influence of the short-range attractive forces diminishes as the density and temperature increases.

Finally, we want to point out that the similar results obtained for the majority of properties by using the full LM1 and LM2 potential models [13] indicate that the discrepancies between the RLM1 and RLM2 results mainly associated with the different effective atomic sizes are balanced by the effects of the strong attractive forces corresponding to the deep potential well of LM1.

\section{ACKNOWLEDGMENTS}

This work was supported by the EEC SCIENCE program under Contract No. SC1-CT91-0754. The financial support from the DGICYT of Spain (Grant No. PB93-0971-C03) is acknowledged.
[1] A. Paskin and A. Rahman, Phys. Rev. Lett. 16, 300 (1966).

[2] D. Schiff, Phys. Rev. 186, 151 (1969).

[3] J. P. Hansen and D. Schiff, Mol. Phys. 25, 1281 (1973).

[4] J. Kushick and B. J. Berne, J. Chem. Phys. 59, 3732 (1973).

[5] S. D. Wijeyesekera and J. Kushick, J. Chem. Phys. 71, 1397 (1979).

[6] D. P. Dean and J. Kushick, J. Chem. Phys. 76, 619 (1982).

[7] J. E. Straub, Mol. Phys. 76, 373 (1992).

[8] S. W. Haan, R. D. Mountain, C. S. Hsu, and A. Rahman, Phys. Rev. A 22, 767 (1980).

[9] S. Kambayashi and Y. Hiwatari, Phys. Rev. E 49, 1251 (1994).

[10] M. M. G. Alemany, C. Rey, and L. J. Gallego, J. Chem. Phys. 105, 8250 (1996).

[11] L. E. González, D. J. González, M. Silbert, and J. A. Alonso, J. Phys. Condens. Matter 5, 4283 (1993).

[12] M. Canales, J. A. Padró, L. E. González, and A. Giró, J. Phys. Condens. Matter 5, 3095 (1993).

[13] M. Canales, L. E. González, and J. A. Padró, Phys. Rev. E 50, 3656 (1994).

[14] H. Sinn and E. Burkel, J. Phys. Condens. Matter 8, 9369 (1996).

[15] M. Canales, A. Giró, and J. A. Padró, J. Non-Cryst. Solids 205-207, 907 (1996).

[16] A. Torcini, U. Balucani, P. H. K. de Jong, and P. Verkerk, Phys. Rev. E 51, 3126 (1995).
[17] D. L. Price, K. S. Singwi, and M. P. Tosi, Phys. Rev. B 2, 2983 (1970).

[18] G. Nowotny, G. Kahl, and J. Hafner, Phys. Scr. 57, 22 (1995).

[19] S. Kambayashi, G. Nowotny, J. Chihara, and G. Kahl, J. NonCryst. Solids 205-207, 914 (1996).

[20] R. D. Mountain, in Proceedings of the Third International Conference on Liquid Metals, Bristol, U.K., 1976, edited by R. Evans and D. A. Greenwood, IOP Conf. Proc. No. 30 (Institute of Physics and Physical Society, London, 1977), p. 62.

[21] U. Balucani, A. Torcini, and R. Vallauri, Phys. Rev. B 47, 3011 (1993).

[22] H. C. Andersen, D. Chandler, and J. D. Weeks, Adv. Chem. Phys. 34, 105 (1976).

[23] J. P. Hansen and I. R. McDonald, Theory of Simple Liquids (Academic, London, 1986).

[24] N. Matsuda, K. Hoshino, and M. Watabe, J. Chem. Phys. 93, 7350 (1990).

[25] J. L. Bretonnet and N. Jakse, Phys. Rev. B 50, 2880 (1994).

[26] M. Canales and J. A. Padró, Mol. Simul. 8, 335 (1992).

[27] U. Balucani and M. Zoppi, Dynamics of the Liquid State (Clarendon, Oxford, 1994).

[28] C. Hoheisel and R. Vogelsang, Comput. Phys. Rep. 8, 1 (1988).

[29] I. Ebbsjö, T. Kinell, and I. Waller, J. Phys. C 13, 1865 (1980). 